\title{
Striving as a nation to become an academic leader in the COVID-19 crisis
}

Ivan Seah, MBBS

\section{INTRODUCTION}

In December 2019, the severe acute respiratory syndrome coronavirus 2 (SARS-CoV-2) emerged from the city of Wuhan. As of 6 March 2020, the virus had spread to more than 80 countries, infecting 98,192 people and causing 3,380 deaths in less than five months. ${ }^{(1)}$

Since the 2003 SARS-CoV epidemic, Singapore's infrastructure has rapidly improved to allow the conduct of high-quality biomedical research. Research facilities such as the Agency for Science, Technology and Research (A*STAR) and Duke-NUS Medical School have been established. The research capabilities of academic hospitals and tertiary institutes have also improved. This has led to the emergence of many local biomedical start-ups and collaborations with international medical device and pharmaceutical companies.

With increased capabilities, Singapore's ability to produce high-quality research on time-sensitive issues, such as those related to the coronavirus disease 2019 (COVID-19), has also improved. Clinical groups from Singapore have recently published in The Lancet and JAMA during the COVID-19 crisis. ${ }^{(2,3)}$ However, more can be done to develop Singapore's attractiveness as a research hub for novel diagnostic and therapeutic technologies for such emerging diseases.

This article will first describe the response of Singapore to the COVID-19 crisis. It will then focus on the various research domains in epidemic settings and highlight the speed at which research is being pursued today. Finally, the article will discuss the current research initiatives and capabilities in Singapore for such research before expounding on the potential improvements that can be implemented.

\section{Singapore's response to the COVID-19 crisis}

On the 23 January 2020, the first case of SARS-CoV-2 in Singapore was reported. ${ }^{(4)}$ As of 6 March, a total of 117 confirmed cases were reported in Singapore with local transmission established. ${ }^{(1)}$ Singapore's early response has been effective at identifying the infected and preventing widespread community transmission. To curb the external sources of infection, bans to entry have been put in place on travellers from Mainland China, Iran, North Italy and South Korea. ${ }^{(5)}$ Local cases were quickly identified with extensive contact tracing, leading to the quarantine of clusters. The infected were given appropriate medical care in tertiary centres. As of 6 March 2020, 82 patients have recovered from the infection. ${ }^{(6)}$ Singapore's response to the epidemic has received praise from international organisations and academics. ${ }^{(7)}$ Most importantly, as of 6 March 2020, there was no mortality among the infected in Singapore. (1) Such evidence highlights Singapore's capability in managing the epidemic.

\section{Research domains in the setting of epidemics}

Epidemics have profound scientific, economic and political implications. From a medical perspective, crucial domains of research include the pathogenesis of aetiologic agents, epidemiological studies, diagnostic technologies and therapeutic modalities.

In the early stages of epidemics, the focus of research is on pathogenesis and epidemiological studies to characterise the aetiologic agent's biological and clinical features. This helps clinicians to recognise patients suspected of harbouring the disease. As more is known about the infective agent, diagnostic methods emerge. The biological and clinical features from previous studies form the basis of diagnostic test development. Further research in this domain will focus on improving test timings, sensitivity and specificity. Research into therapeutic modalities and vaccinations usually develop later, as they require a large amount of coordination between governments, hospitals and industry. Research on emerging infectious diseases is key, as it aids in the early recognition of the disease and improves transmission prevention measures. Most importantly, the morbidity and mortality of the disease can be reduced as well.

The speed of research has improved tremendously as compared with that of the SARS-CoV crisis eight years ago. In this COVID-19 crisis, the genome of the SARS-CoV-2 virus was sequenced and released within a month from the start of the epidemic, facilitating the creation of diagnostic tests. ${ }^{(8)}$ In comparison, the causative agent of SARS-CoV was identified almost six months after the first case. ${ }^{(9)}$ Investigations of therapeutic options have also been accelerated. Remdesivir, an adenosine nucleotide analogue produced by Gilead Sciences, started two Phase III studies just two months after the start of the epidemic in China (ClinicalTrials. gov NCT04257656, NCT04252664). Such breakneck speeds are possible due to not only technological advancements, but also improved processes of regulatory agencies globally.

Research in the epidemic setting is a relevant pursuit for Singapore for various reasons. Firstly, clinical research can provide crucial information on clinical scenarios, which are particularly relevant in the Singapore context. For instance, a recent paper

${ }^{1}$ Department of Ophthalmology, National University Hospital, Singapore

Correspondence: Dr Ivan Seah, Medical Officer, Department of Ophthalmology, National University Hospital, 1E Kent Ridge Road, NUHS Tower Block, Level 7, Singapore 119228. ivanseah22031991@gmail.com 
published in The Lancet Infectious Diseases highlighted a case of COVID-19 that masqueraded as a false-positive dengue fever serology test. ${ }^{(10)}$ This alerted primary care physicians to be aware of mislabelling patients as having dengue fever during the crisis. Secondly, research into diagnostics can allow the country to be self-sustaining. Even though the diagnostic technology may have been developed in countries that experienced the crisis earlier than Singapore, such technology may not be available here due to logistical reasons. As such, the pursuit of epidemic research is still extremely relevant for Singapore.

\section{Singapore's research efforts in the COVID-19 crisis}

Since the start of the COVID-19 crisis, government agencies have been providing financial support for COVID-19-related research. Individual hospitals such as the National University Hospital, Singapore General Hospital and Tan Tock Seng Hospital have internal grant calls. On a national scale, the National Medical Research Council has launched a COVID-19 research fund, which provides a quantum of up to SGD 1 million over a period of one year for research in transmission, pathogenesis, serological testing and modelling of the disease. ${ }^{(11)}$

Singapore's research efforts have culminated in epidemiological studies published in reputed journals. In an article by the National Centre for Infectious Diseases published in JAMA, a case series of 18 patients diagnosed with SARSCoV-2 was described. ${ }^{(3)}$ The Lancet also published a case series of two patients with false-positive results from dengue rapid serological testing, who were later confirmed to have the SARSCoV-2 infection. ${ }^{(10)}$ A group from Duke-NUS Medical School also established themselves as the first in the world to utilise serological testing for contact tracing. ${ }^{(12)}$

\section{Improvements to accelerate development of diagnostic tools and therapeutics}

However, research into new diagnostic methods and therapeutics are only starting to take off. A brief search of the ClinicalTrials. gov database on 7 March 2020 saw 46 relevant interventional COVID-19 clinical trials currently being conducted in mainland China, the United States, Hong Kong and Canada. Singapore has no registered trials. ${ }^{(13)}$ Based on the chronology of spread, it is surprising that countries that were inflicted with the disease at later dates have established clinical trials earlier than Singapore. In Singapore, the patient pool and state-of-the-art medical facilities provide a good setting for clinical trials to generate reliable data. Given the infrastructure and financial support available, Singapore should leverage on these resources to be at the forefront of diagnostics and therapeutics development.

To be successful in the pursuit of these research domains, improvements in three aspects should be considered: (1) establishing shared viral stock or reagents for testing of hypotheses; (2) organising platforms for multidisciplinary collaborations; and (3) making ethics and regulatory processes dynamic and transparent.

While research into infective agents can be potentially dangerous, it should not prevent academic groups from venturing into such fields. Rather, research should be conducted with care to prevent transmission. Academic groups in Singapore should be able to gain access to resources such as the recombinant receptor-binding domain proteins, genomic RNA and isolates of the SARS-CoV-2 via a central agency. This central agency should allocate such resources based on an academic group's scientific proficiency and infrastructural capabilities of conducting research in a biosafety level 2 or 3 facility. The Biodefense and Emerging Infections Research Resources Repository, established by the National Institute of Allergy and Infectious Diseases in America, utilises this model to ensure efficient and safe delivery of such resources. This enables a large variety of academic groups to partake in such research, allowing the development of unique perspectives regarding the virus.

Diagnostic tools and therapeutic modalities are extremely complex research fields that usually require multidisciplinary input. Scientists, clinicians and industry experts often have to exchange ideas and resources to successfully produce translatable technology. On 11-12 February 2020, the World Health Organization conducted the COVID-19 Global Research and Innovation Forum. The event brought together over 300 scientists, researchers and national public health experts to discuss research priorities and share scientific data, accelerating the generation of critical research. ${ }^{(14)}$ Singapore should consider organising such platforms to foster crucial collaborations. It is also important for Singapore to seek collaboration opportunities with industry partners through the close relationships fostered prior to the epidemic, including those with medical device and large pharmaceutical companies. Such collaborations should focus on developing synergy between Singapore research groups and large companies. Having published in various globally recognised journals, Singapore's research groups can provide novel solutions to the problems faced during epidemics, while large pharmaceutical and medical device companies can provide the manufacturing expertise to accelerate the development of feasible products. Alliances with local medical and biotechnology start-ups should also not be forgotten. With the increasing number of such start-ups in Singapore, properly selected collaborations can provide novel approaches to curbing the epidemic.

Finally, research ethics and regulatory boards should adopt a dynamic approach to regulating the approval of novel diagnostic tools and therapeutics for trial purposes. In a time-sensitive epidemic, it is key to maintain a balance between speed of approval and safety of a clinical trial material. In the epidemic setting, the institutional review boards of healthcare institutions and the Health Sciences Authority can consider developing a fast-track pathway for clinical trial material approval, contingent to trial materials fulfilling certain criteria such as favourable pre-clinical results. Most importantly, after developing these pathways, the roadmaps should be made publicly available so that both local and multinational companies will understand the potential hurdles to approval, guiding their decisions to conduct clinical trials in Singapore. In addition, research ethics and regulatory boards should aim to ensure that studies can be conducted in not only a safe but also timely manner. 


\section{CONCLUSION}

The ability of Singapore to conduct biomedical research has improved tremendously over the past decade. By leveraging on the improved infrastructure and close teamwork with research ethics and regulatory boards, the COVID-19 epidemic may be an opportunity for this nation to rise as an academic leader.

\section{REFERENCES}

1. World Health Organization. Coronavirus disease 2019 (COVID-19) Situation Report - 46. 6 March 2020. Available at: https://www.who.int/docs/ default-source/coronaviruse/situation-reports/20200306-sitrep-46-covid-19. pdf?sfvrsn=96b04adf_4. Accessed March 12, 2020.

2. Ong SWX, Tan YK, Chia PY, et al. Air, surface environmental, and personal protective equipment contamination by severe acute respiratory syndrome coronavirus 2 (SARS-CoV-2) from a symptomatic patient. JAMA 2020; 20:536.

3. Young BE, Ong SWX, Kalimuddin S, et al. Epidemiologic features and clinical course of patients infected with SARS-CoV-2 in Singapore. JAMA 2020; 323:1488-94.

4. Abdullah Z, Salamat H. Singapore confirms first case of Wuhan virus. In: Channel NewsAsia. Available at: https://www.channelnewsasia.com/news/singapore/ wuhan-virus-pneumonia-singapore-confirms-first-case-12312860. Accessed February 14, 2020

5. COVID-19: Travel restrictions for foreign visitors entering Singapore. In: gov sg [online]. Available at: https://www.gov.sg/article/covid-19-travel-restrictionsfor-foreign-visitors-entering-singapore. Accessed March 27, 2020.
6. Ministry of Health, Singapore. One more case discharged; thirteen new cases of COVID-19 infection confirmed. Available at: https://www.moh.gov.sg/ news-highlights/details/one-more-case-discharged-thirteen-new-cases-of-covid19-infection-confirmed.

7. WHO impressed by how Singapore handles coronavirus outbreak. The Straits Times 2020 Feb 20. Available at: https://www.straitstimes.com/singapore/whoimpressed-by-how-spore-handles-outbreak. Accessed February 26, 2020.

8. Kupferschmidt K. 'A completely new culture of doing research.' Coronavirus outbreak changes how scientists communicate. Science 2020 Feb 26. https:// doi.org/10.1126/science.abb4761. [Epub ahead of print]

9. World Health Organization. Update 95 - SARS: Chronology of a serial killer. Available at: https://www.who.int/csr/don/2003_07_04/en/.

10. Yan G, Lee CK, Lam LTM, et al. Covert COVID-19 and false-positive dengue serology in Singapore. Lancet Infect Dis 2020; 20:536.

11. National Medical Research Council, Ministry of Health, Singapore. The COVID-19 Research Fund is provided to support COVID-19-related research. Available at: https://www.nmrc.gov.sg/grants/competitive-research-grants/ covid-19-research-fund.

12. Normile D. Singapore claims first use of antibody test to track coronavirus infections. Science 2020 Feb 27. https://doi.org/10.1126/science.abb4942.

13. Search results for interventional studies and coronavirus. In: ClinicalTrials.gov. Available at: https://clinicaltrials.gov/ct2/results?cond=coronavirus\&Search=Apply\&age_ $\mathrm{v}=$ \&gndr=\&type $=$ Intr\&rslt $=$. Accessed March 7, 2020.

14. World Health Organization. COVID-19 Public Health Emergency of International Concern (PHEIC). Global research and innovation forum: towards a research roadmap. Available at: https:/www.who.int/blueprint/priority-diseases/ key-action/Global_Research_Forum_FINAL_VERSION_for_web_14_feb_2020. pdf?ua $=11$. 